\title{
Unraveling the Breast: Advances in Mammary Biology and Cancer Methods
}

\author{
Teresa Monkkonen $^{1}$ (D) Gunnhildur Ásta Traustadóttir ${ }^{2}$ (D) $\cdot$ Zuzana Koledova $^{3}$ (i)
}

Received: 14 December 2020 / Accepted: 20 December 2020 / Published online: 21 January 2021

(c) The Author(s), under exclusive licence to Springer Science+Business Media, LLC part of Springer Nature 2021

\begin{abstract}
The field of mammary gland biology and breast cancer research encompasses a wide range of topics and scientific questions, which span domains of molecular, cell and developmental biology, cancer research, and veterinary and human medicine, with interdisciplinary overlaps to non-biological domains. Accordingly, mammary gland and breast cancer researchers employ a wide range of molecular biology methods, in vitro techniques, in vivo approaches as well as in silico analyses. The list of techniques is ever-expanding; together with the refinement of established, staple techniques in the field, new technologies keep emerging thanks to technological advances and scientific creativity. This issue of the Journal of Mammary Gland Biology and Neoplasia represents a compilation of original articles and reviews focused on methods used in mammary gland biology and breast cancer research.
\end{abstract}

Keywords Breast $\cdot$ Database $\cdot$ Exosomes $\cdot$ Heterogeneity $\cdot$ Mammary gland $\cdot$ Milk cells $\cdot$ Multiplexing $\cdot$ Organoid

\section{Editorial}

The signature organ of mammals, the mammary gland, serves one specific function-to secrete milk to nourish the offspring. Besides the basic nutritional components, such as water, carbohydrates, proteins and fats, milk also contains cells, whose characteristics, origin and function have not been fully elucidated. Two articles in this issue aimed to further characterize these cells in human milk. SchultzPernice and colleagues [1] performed a morphological analysis of membrane-enclosed structures in human milk, and explored different methods for enriching cells in milk as well as reducing the presence of milk fat globules. The authors found a variety of epithelial cell types in human milk, including secretory binucleated epithelial cells. Martin-Carli and colleagues [2] report on their method

Zuzana Koledova

koledova@med.muni.cz

1 Department of Pathology, University of California, San Francisco, USA

2 Stem Cell Research Unit, Department of Anatomy, Faculty of Medicine, School of Health Sciences, Biomedical Center, University of Iceland, Reykjavík, Iceland

3 Department of Histology and Embryology, Faculty of Medicine, Masaryk University, Brno, Czech Republic for analyzing human milk samples using single cell RNA sequencing. The authors present data on the efficacy of cryopreserving cells from milk and subsequent fluorescenceactivated cell sorting, the results from the analysis of the molecular signatures of cells in milk, as well as insights into biases of this approach (for example, the low abundance of EPCAM+cells). Their findings on human milk-derived cell sub-populations and their potential functional relationships give rise to interesting biological questions, such as the mechanisms regulating triglyceride versus de novo fatty acid synthesis. Moreover, it will be interesting to integrate the data from this study with emerging studies from other cohorts, such as the study of Twigger and colleagues [3], and to decipher the differentiation programs for luminal cells, metabolism, and breast cancer risk associated with lactation.

The lactational capacity of the mammary gland depends on proper organ development and is under tight, multigenic control. Genetically engineered mouse models (GEMMs) have provided invaluable insights into the pathways involved in the control of mammary gland development and function. In their review, Stewart and Davis [4] provide a useful manual for analyzing mammary gland developmental phenotypes and lactational defects in GEMMs, including histological techniques, preparation of tissue lysates, and milk collection. Moreover, they provide useful tips on proper mouse husbandry and mating while highlighting the 
importance of details such as litter standardization, and then discuss the caveats of inducible mouse models.

Upon pregnancy, the mammary gland undergoes substantial tissue remodeling, accompanied by epigenetic changes in mammary epithelial cells [5]. In their article, Ciccone and colleagues [6] present the validation of a $3 \mathrm{D}$ organoid culture method that can be used to mimic responses by the mammary epithelium to hormones of pregnancy. Their method based on primary mammary epithelial organoids is similar to that recently developed by Sumbal, Chiche and colleagues [7], and bears the potential for dissecting pregnancy-induced effects on the epigenome and transcriptional output of mammary epithelial cells.

Mammary organoids and 3D cell cultures alike have been a staple in the toolbox of mammary gland and breast cancer researchers for decades because of their superior ability to faithfully recapitulate in vitro the diverse aspects of tissue architecture, spatial complexity, and heterogeneity found in vivo. The review article by Sumbal and colleagues [8] provides an overview of mammary 3D cell culture techniques, discusses their advantages, shortcomings and current challenges, and brings forward recent attempts to reconstruct the complex mammary gland microenvironment in vitro and ex vivo. The review summarizes multiple studies that employed 3D cell culture and organoid techniques to decipher events during mammary gland development, lactation, and cancer. Furthermore, the authors discuss synergy between the use of mouse models and organoids, and techniques for imaging 3D cell cultures.

Compared to 2D cultures, one of the shortcomings of $3 \mathrm{D}$ cell cultures is that the 3D cultures are more expensive and laborious. To overcome these limitations, Wrenn and colleagues [9] developed a suspension culture method optimized for production of large numbers of organoids, enabling impressive expansion from one million to over 100 million cells from mouse-derived tumors within two weeks. Moreover, building on their protocol, they established conditions for efficient lentiviral transduction to generate organoids having genetic knockout, knockdown, or overexpression. This method will enable researchers to make the best use of pre-clinical models, save time and costs in labs impacted by COVID-19 slowdowns, and increase the feasibility of biochemical follow-up relative to other methods.

Efficient viral transduction of mammary cells is the central focus of two articles in this issue. Lan and Mikkola [10] provide a protocol for transducing embryonic mammary gland explants using adeno-associated viruses (AAVs). Building on their expertise in embryonic mammary gland dissection, epithelium-mesenchyme separation, and culture, they optimized crucial steps for AAV infection of the whole explant or isolated mammary epithelial buds. Moreover, they report on important differences in preferential targeting of epithelial and mesenchymal compartments by different serotypes of the AAVs that need to be considered by researchers interested in genetic modification of the embryonic mammary gland ex vivo using this protocol. Furthermore, $\mathrm{Bu}$ and $\mathrm{Li}$ [11] describe a procedure for transducing rat mammary epithelial cells in vivo using intraductal injection of lentivirus. This method enables stable introduction of marker genes or oncogenes of interest. The oncogene-transduced cells can generate mammary tumors in rats quickly, including estrogen receptor positive $(E R+)$ tumors, which are difficult to model in mice. Thus, this method provides a useful tool for breast cancer research, especially for ER + and hormone-dependent tumors.

Obesity is an acknowledged risk factor for the development of hormone-receptor positive breast tumors in postmenopausal women (reviewed in [12]). In their review, Giles and Wellberg [13] draw on their own experience and discuss how to generate preclinical rodent models that integrate obesity and simulated menopause with the development of hormonereceptor positive tumors. These authors describe how to promote and measure obesity in rodents, the various obesity models that are available, and how metabolic health may be an important contributor to obesity-associated cancer. The authors also highlight the importance of diet selection and housing temperature on the promotion of obesity in rodents and discuss approaches to model menopause in rodents and the importance of estrogen supplementation for establishment of estrogen-responsive tumors.

Exosomes, the nano-sized vesicles capable of mediating intercellular communication over short and long distances with a putative role in cancer progression (reviewed in [14]), have recently gained increased attention in breast cancer. Pelissier Vatter and colleagues [15] provide an overview of various methods for isolating and characterizing exosomes. In their review, they discuss human and mouse models of breast cancer exosomes, how exosomes can be labelled to assess organ distribution, and how the effect of exosomes on target cells can be experimentally evaluated in vitro and in vivo.

Breast cancer is a heterogeneous disease that differs greatly between patients (both intertumoral and interpatient heterogeneity). Even within a tumor, the cells show substantial diversity in somatic mutations, gene expression, proteomic and metabolic programming (intratumoral heterogeneity) [16]. This intratumor heterogeneity represents a major obstacle for breast cancer diagnosis, prognosis and effective therapeutic intervention, and has therefore been the subject of increasing research efforts. Accordingly, the number of articles on intratumor heterogeneity has increased from tens to hundreds per year since 2010. To highlight this issue, the review by Baek and colleagues [17] compares the ability of different methods to capture breast tumor heterogeneity and discusses the promise and utility of liquid biopsy. They also address 
key findings regarding clonal evolution and therapeutic resistance from studies of breast tumor heterogeneity, and provide a helpful perspective for this growing and evolving field.

Interactions between tumor cells and the immune system play a crucial role in tumor progression [18]. Immune cells can be involved in both pro- and anti-tumorigenic activities, and successful therapeutic intervention requires identification of immune cell activities with human tumors. To this end, Mori and colleagues [19] optimized tyramide-based signal amplification for multiplex immunohistochemistry to analyze immune cells in tissue samples. To increase its reproducibility, the staining is automated using a slide stainer, followed by slide scanning and image analysis. This stands to be a useful method for clinical practice to assess immune cell activities during patient treatment regimens as well as in breast cancer research.

The advance of techniques for analyzing whole genomes, epigenomes, transcriptomes, proteomes, and metabolomes has brought with it a tremendous amount of data that is deposited in a range of databases, and at the fingertips of researchers. This provides the immense potential for scientists to boost their studies, as long as they have the skills and expertise to work with such databases. In their timely article, van de Grift and colleagues [20] share useful tips for mining large "omics" datasets using online tools. Using the example of the $W n t 7 b$ gene, the authors demonstrate how these tools can be used to generate new hypotheses and answer biological questions in silico.

In summary, this exciting special issue on methods includes original research articles alongside detailed protocols and reviews. Combined, they all stand to improve our understanding of different techniques, while helping with method selection, increasing technical skills, and promoting reproducibility to advance mammary gland and breast cancer research. We believe the content of this special issue will be of widespread interest and utility to the community of mammary gland biology and breast cancer scientists.

Funding The authors of this work were supported by grants from the American Cancer Society-Jean Perkins Foundation Postdoctoral Fellowship PF-18-227-01-CSM (T.M.), The Science Fund of the Icelandic Cancer Society (G.A.T.), the Grant Agency of Masaryk University (Z.K., project no. MUNI/G/1446/2018), and the Faculty of Medicine MU to junior researcher (Z.K., ROZV/28/LF/2020).

\section{Compliance with Ethical Standards}

Conflict of Interest The authors declare that they have no conflict of interest.

\section{References}

1. Schultz-Pernice I, Engelbrecht LK, Petricca S, Scheel CH, Twigger A-J. Morphological Analysis of Human Milk Membrane Enclosed Structures Reveals Diverse Cells and Cell-like Milk Fat Globules. J Mammary Gland Biol Neoplasia. 2020. https://doi. org/10.1007/s10911-020-09472-1.

2. Martin Carli JF, Trahan GD, Jones KL, et al. Single Cell RNA Sequencing of Human Milk-Derived Cells Reveals Sub-Populations of Mammary Epithelial Cells with Molecular Signatures of Progenitor and Mature States: a Novel, Non-invasive Framework for Investigating Human Lactation Physiology. J Mammary Gland Biol Neoplasia. 2020. https://doi.org/10.1007/s10911-020-09466-z.

3. Twigger A-J, Engelbrecht LK, Bach K, Schultz-Pernice I, Petricca $\mathrm{S}$, Scheel $\mathrm{CH}$, et al. Transcriptional changes in the mammary gland during lactation revealed by single cell sequencing of cells from human milk. bioRxiv. 2020;2020.11.06.371443.

4. Stewart TA, Davis FM. Got Milk? Identifying and Characterizing Lactation Defects in Genetically-Engineered Mouse Models. J Mammary Gland Biol Neoplasia. 2020. https://doi.org/10.1007/ s10911-020-09467-y.

5. Feigman MJ, Moss MA, Chen C, Cyrill SL, Ciccone MF, Trousdell $\mathrm{MC}$, et al. Pregnancy reprograms the epigenome of mammary epithelial cells and blocks the development of premalignant lesions. Nat Commun. 2020;11:2649.

6. Ciccone MF, Trousdell MC, dos Santos CO. Characterization of Organoid Cultures to Study the Effects of Pregnancy Hormones on the Epigenome and Transcriptional Output of Mammary Epithelial Cells. J Mammary Gland Biol Neoplasia. 2020. https:// doi.org/10.1007/s10911-020-09465-0.

7. Sumbal J, Chiche A, Charifou E, Koledova Z, Li H. Primary Mammary Organoid Model of Lactation and Involution. Front Cell Dev Biol [Internet]. Frontiers; 2020 [cited 2020 Apr 5];8. Available from: https://www.frontiersin.org/articles/ https://doi. org/10.3389/fcell.2020.00068/full.

8. Sumbal J, Budkova Z, Traustadóttir GÁ, et al. Mammary Organoids and 3D Cell Cultures: Old Dogs with New Tricks. J Mammary Gland Biol Neoplasia. 2020. https://doi.org/10.1007/ s10911-020-09468-x.

9. Wrenn ED, Moore BM, Greenwood E, et al. Optimal, Large-Scale Propagation of Mouse Mammary Tumor Organoids. J Mammary Gland Biol Neoplasia. 2020. https://doi.org/10.1007/s10911-02009464-1.

10. Lan Q, Mikkola ML. Protocol: Adeno-Associated Virus-Mediated Gene Transfer in Ex Vivo Cultured Embryonic Mammary Gland. J Mammary Gland Biol Neoplasia. 2020. https://doi.org/10.1007/ s10911-020-09461-4.

11. $\mathrm{Bu} \mathrm{W,} \mathrm{Li} \mathrm{Y.} \mathrm{Intraductal} \mathrm{Injection} \mathrm{of} \mathrm{Lentivirus} \mathrm{Vectors} \mathrm{for} \mathrm{Stably}$ Introducing Genes into Rat Mammary Epithelial Cells in Vivo. J Mammary Gland Biol Neoplasia. 2020. https://doi.org/10.1007/ s10911-020-09469-w.

12. Hillers-Ziemer LE, Arendt LM. Weighing the Risk: effects of Obesity on the Mammary Gland and Breast Cancer Risk. J Mammary Gland Biol Neoplasia. 2020;25:115-31.

13. Giles ED, Wellberg EA. Preclinical Models to Study Obesity and Breast Cancer in Females: Considerations, Caveats, and Tools. J Mammary Gland Biol Neoplasia. 2020. https://doi.org/10.1007/ s10911-020-09463-2.

14. Wortzel I, Dror S, Kenific CM, Lyden D. Exosome-Mediated Metastasis: Communication from a Distance. Dev Cell. 2019;49:347-60.

15. Pelissier Vatter FA, Lucotti S, Zhang H. Recent Advances in Experimental Models of Breast Cancer Exosome Secretion, Characterization and Function. J Mammary Gland Biol Neoplasia. 2020. https://doi.org/10.1007/s10911-020-09473-0. 
16. Turashvili G, Brogi E. Tumor Heterogeneity in Breast Cancer. Front Med (Lausanne) [Internet]. 2017 [cited 2020 Dec 7];4. Available from: https://www.ncbi.nlm.nih.gov/pmc/articles/ PMC5727049/

17. Baek M, Chang JT, Echeverria GV. Methodological Advancements for Investigating Intra-tumoral Heterogeneity in Breast Cancer at the Bench and Bedside. J Mammary Gland Biol Neoplasia. 2020. https://doi.org/10.1007/s10911-020-09470-3.

18. Swann JB, Smyth MJ. Immune surveillance of tumors. J Clin Invest. 2007;117:1137-46.

19. Mori H, Bolen J, Schuetter L, Massion P, Hoyt CC, VandenBerg $\mathrm{S}$, Esserman L, Borowsky AD, Campbell MJ. Characterizing the tumor immune microenvironment with tyramide based multiplex immunofluorescence. J Mammary Gland Biol Neoplasia. 2021.

20. van de Grift YBC, Heijmans N, van Amerongen R. How to Use Online Tools to Generate New Hypotheses for Mammary Gland Biology Research: A Case Study for Wnt7b. J Mammary Gland Biol Neoplasia. 2020. https://doi.org/10.1007/s10911-020-09474-z.

Publisher's Note Springer Nature remains neutral with regard to jurisdictional claims in published maps and institutional affiliations. 